\title{
ON A UNIFORM BOUND FOR THE NUMBER OF EXCEPTIONAL LINEAR SUBVARIETIES IN THE DYNAMICAL MORDELL-LANG CONJECTURE
}

\author{
Joseph H. Silverman And Bianca Viray
}

\begin{abstract}
Let $\phi: \mathbb{P}^{n} \rightarrow \mathbb{P}^{n}$ be a morphism of degree $d \geq 2$ defined over $\mathbb{C}$. The dynamical Mordell-Lang conjecture says that the intersection of an orbit $\mathcal{O}_{\phi}(P)$ and a subvariety $X \subset \mathbb{P}^{n}$ is usually finite. We consider the number of linear subvarieties $L \subset \mathbb{P}^{n}$ such that the intersection $\mathcal{O}_{\phi}(P) \cap L$ is "larger than expected." When $\phi$ is the $d^{\text {th }}$-power map and the coordinates of $P$ are multiplicatively independent, we prove that there are only finitely many linear subvarieties that are "super-spanned" by $\mathcal{O}_{\phi}(P)$, and further that the number of such subvarieties is bounded by a function of $n$, independent of the point $P$ and the degree $d$. More generally, we show that there exists a finite subset $S$, whose cardinality is bounded in terms of $n$, such that any $n+1$ points in $\mathcal{O}_{\phi}(P) \backslash S$ are in linear general position in $\mathbb{P}^{n}$.
\end{abstract}

\section{The dynamical Mordell-Lang conjecture}

The classical Mordell conjecture says that a curve $C$ of genus $g \geq 2$ defined over a number field $K$ has only finitely many $K$-rational points. One may view $C$ as embedded in its Jacobian $J$, and then Mordell's conjecture may be reformulated as saying that $C$ intersects the finitely generated group $J(K)$ in only finitely many points. Taking this viewpoint, Lang conjectured that if $\Gamma \subset A$ is a finitely generated subgroup of an abelian variety $A$ and if $X \subset A$ is a subvariety of $A$, then $X \cap \Gamma$ is contained in a finite union of translates of proper abelian subvarieties of $A$. The Mordell-Lang conjecture for abelian varieties was proven by Faltings [8, 9], building on ideas pioneered by Vojta [18] in his alternative proof of the original Mordell conjecture.

The classical Mordell-Lang conjecture may be reformulated in dynamical terms as follows. Let $P_{1}, \ldots, P_{r}$ be generators of $\Gamma$, and for each $1 \leq i \leq r$, let $T_{i}: A \rightarrow A$ be the translation-by- $P_{i}$ map, i.e., $T_{i}(Q)=Q+P_{i}$. Further let $\mathcal{T}$ be the group of self-maps of $A$ generated by $T_{1}, \ldots, T_{r}$. Then $\Gamma$ is simply the complete orbit of 0 by the group of maps $\mathcal{T}$, so the Mordell-Lang conjecture is a statement about the intersection of an orbit and a subvariety.

The following is a dynamical analogue of the Mordell-Lang conjecture for selfmorphisms of algebraic varieties; see [4, 10].

Conjecture 1.1 (Dynamical Mordell-Lang Conjecture). Let $\phi: V \rightarrow V$ be a selfmorphism of an algebraic variety defined over $\mathbb{C}$, let $X \subset V$ be a subvariety, and let $P \in V(\mathbb{C})$. Then

$$
\left\{n \geq 0: \phi^{n}(P) \in X\right\}
$$

Received by the editors 10 September, 2011.

1991 Mathematics Subject Classification. Primary: 37P15; Secondary: 11D45, 37P05.

Key words and phrases. arithmetic dynamics, Mordell-Lang conjecture. 
is a finite union of arithmetic progressions (where a single integer is viewed as an arithmetic progression with common difference 0 ).

There are currently only a few scattered results in the literature related to the Mordell-Lang conjecture in the dynamical setting. These include results on étale maps, an analogue for Drinfeld modules, and results for maps of various special types, for example diagonal maps $\phi\left(z_{1}, \ldots, z_{n}\right)=\left(f_{1}\left(z_{1}\right), \ldots, f_{n}\left(z_{n}\right)\right)$; see $[2,3,10,11,12]$.

Write

$$
\mathcal{O}_{\phi}(P)=\left\{\phi^{n}(P): n \geq 0\right\}
$$

for the forward orbit of $P$. The intersection $X \cap \mathcal{O}_{\phi}(P)$ may be infinite if there is some positive-dimensional subvariety $Y \subset X$ that is periodic, i.e., $\phi^{N}(Y)=Y$, since then $\phi^{k}(P) \in Y$ implies that $\phi^{k+i N}(P) \in Y$ for all $i \geq 0$. If there is no such subvariety, then one generally expects the intersection $X \cap \mathcal{O}_{\phi(P)}$ to be finite.

We now turn the Mordell-Lang problem around and consider the set of subvarieties $X$ whose intersection with $\mathcal{O}_{\phi}(P)$ is finite, but "larger than one would expect." In this paper we restrict attention to self-maps of $\mathbb{P}^{n}$ and linear subspaces $X$, which still present sufficiently many difficulties to merit study. For example, if $\phi: \mathbb{P}^{2} \rightarrow \mathbb{P}^{2}$, then it seems plausible that there should be only finitely many lines in $\mathbb{P}^{2}$ that contain three (or more) points of the orbit $\mathcal{O}_{\phi}(P)$. More generally, one might expect that there are only finitely many hyperplanes $H$ in $\mathbb{P}^{n}$ that contain $n+1$ points of the orbit $\mathcal{O}_{\phi}(P)$, but this is not quite true. The problem is that there might be some lower dimensional linear space $L \subset \mathbb{P}^{n}$ that contains $n$ points of $\mathcal{O}_{\phi}(P)$, and then every hyperplane $H$ containing $L$ and having non-empty intersection with $\mathcal{O}_{\phi}(P) \backslash L$ will $a$ fortiori contain $n+1$ points of $\mathcal{O}_{\phi}(P)$. The solution is to look only at hyperplanes $H$ containing $n+1$ points $Q_{0}, \ldots, Q_{n}$ of $\mathcal{O}_{\phi}(P)$ such that $H$ is spanned by every subset of $\left\{Q_{0}, \ldots, Q_{n}\right\}$ consisting of $n$ points.

We will say that an $(r-1)$-dimensional linear space $L \subset \mathbb{P}^{n}$ is super-spanned by the set of points $\left\{Q_{0}, \ldots, Q_{r}\right\}$ if every subset consisting of $r$ points spans $L$. With this definition, we can state our main conjecture.

Conjecture 1.2. Let $\phi: \mathbb{P}^{n} \rightarrow \mathbb{P}^{n}$ be a morphism of degree $d \geq 2$ defined over $\mathbb{C}$, and let $P \in \mathbb{P}^{n}(\mathbb{C})$ be a point whose orbit $\mathcal{O}_{\phi}(P)$ is Zariski dense in $\mathbb{P}^{n}$. Let $r \geq 1$. Then there are only finitely many linear subspaces $L \subset \mathbb{P}^{n}$ of dimension $r-1$ such that $L \cap \mathcal{O}_{\phi}(P)$ contains a set of $r+1$ points that super-spans $L$. Furthermore, the number of such linear subspaces can be bounded by a function that depends only on $n$ and $d$.

Conjecture 1.2 may be viewed as saying that the orbit $\mathcal{O}_{\phi(P)}$ is "almost" in linear general position in the following sense: after removing finitely many linear subspaces of $\mathbb{P}^{n}$, no $r+2$ points of $\mathcal{O}_{\phi(P)}$ are contained in any of the remaining $(r-1)$ dimensional linear spaces, and moreover, that the number of linear subspaces that need to be removed is bounded by a function that depends only on $n$ and $d$. If, in addition, one knows that $L \cap \mathcal{O}_{\phi}(P)$ is finite for all linear spaces $L$, then one gets a stronger version of "almost" in linear general position, i.e., there exists a finite subset $S \subseteq \mathcal{O}_{\phi}(P)$, whose cardinality is bounded in terms of $n$ and $d$, such that any $n+1$ points in $\mathcal{O}_{\phi}(P) \backslash S$ are in linear general position in $\mathbb{P}^{n}$. 
In full generality, Conjecture 1.2 seems difficult. The primary result in this paper is a proof of the conjecture for the $d^{\text {th }}$-power map, under the (possibly) weaker assumption that the coordinates of the point $P$ are multiplicatively independent. In this case, we are able to prove a uniform bound that is independent of both $P$ and $d$.

Theorem 1.3. Let $d \geq 2$, let

$$
\phi\left(\left[z_{0}, \ldots, z_{n}\right]\right)=\left[z_{0}^{d}, \ldots, z_{n}^{d}\right]
$$

be the $d^{\text {th }}$-power map on $\mathbb{P}^{n}$, and let $P \in \mathbb{P}^{n}(\mathbb{C})$ be a point whose coordinates are non-zero and multiplicatively independent. Then Conjecture 1.2 is true for $\phi$ and $P$. More precisely, the number of super-spanned linear subspaces $L$ is bounded solely in terms of $n$, independent of the point $P$ and the degree $d$.

The proof of Theorem 1.3 can be adapted to prove the following uniform bound for the number of points in $\mathcal{O}_{\phi}(P) \cap L$. We give the proof of Corollary 1.4 in Section 5 .

Corollary 1.4. Let $\phi$ and $P$ be as in the statement of Theorem 1.3. Then for any linear subspace $L$, the intersection $\mathcal{O}_{\phi}(P) \cap L$ is finite, and its size is bounded solely in terms of $n$, independent of $P$ and $d$.

Remark 1.5. In the setting of Conjecture 1.2, the finiteness of the set $L \cap \mathcal{O}_{\phi}(P)$ follows trivially from the dynamical Mordell-Lang conjecture (Conjecture 1.1). To see this, suppose that $\#\left(L \cap \mathcal{O}_{\phi}(P)\right)=\infty$. Then Conjecture 1.1 says that $\{n \geq 0$ : $\left.\phi^{n}(P) \in L\right\}$ contains a non-trivial arithmetic progression $q \mathbb{N}+r$, from which we find that

$$
\begin{aligned}
\mathcal{O}_{\phi}(P) & =\left\{P, \phi(P), \ldots, \phi^{r-1}(P)\right\} \cup \bigcup_{i=r}^{q+r-1} \phi^{i-r}\left(\left\{\phi^{j q+r}(P): j \geq 0\right\}\right) \\
& \subset\left\{P, \phi(P), \ldots, \phi^{r-1}(P)\right\} \cup \bigcup_{i=r}^{q+r-1} \phi^{i-r}(L),
\end{aligned}
$$

contradicting the assumption that $\mathcal{O}_{\phi}(P)$ is Zariski dense. Assuming the finiteness of $L \cap \mathcal{O}_{\phi}(P)$ for all $L$, we note that Conjecture 1.2 and an easy induction on $\operatorname{dim} L$ can be used to bound $\#\left(L \cap \mathcal{O}_{\phi}(P)\right)$ in terms of $n$ and $d$.

We conclude this introduction by giving a brief overview of the key steps in the proof of Theorem 1.3. We consider $r+1$ arbitrary iterates of $\phi$ applied to $P$, say

$$
Q_{0}=\phi^{m_{0}}(P), \quad Q_{1}=\phi^{m_{1}}(P), \ldots, Q_{r}=\phi^{m_{r}}(P),
$$

and we assume that $Q_{0}, \ldots, Q_{r}$ super-span a linear subspace $L$ of dimension $r$. The fact that these points lie in $L$ means that the $(r+1)$-by- $(n+1)$ matrix $A$ whose rows are the points $Q_{0}, \ldots, Q_{r}$ has rank $r$. Hence all of its $(r+1)$-by- $(r+1)$ minors have zero determinant, and expanding these determinants as sums over the permutation group $\mathcal{S}_{r+1}$ gives various linear combinations of products of powers of the coordinates of the point $P$. We then apply a deep theorem on uniform bounds for the number of non-degenerate solutions to $S$-unit equations $u_{1}+\cdots+u_{N}=0$ due to Evertse et al. [7]. If none of the subsums over subsets of $\mathcal{S}_{r+1}$ vanishes, the proof is essentially complete. (This is where we use the multiplicative independence of the coordinates to $P$, since what we really get is that certain products of powers of the coordinates take on only finitely values.) However, it is certainly possible for subsums of the determinant 
sums to vanish, so we need to consider all possible partitions of $\mathcal{S}_{r+1}$ associated to vanishing subsums. This leads to a fairly elaborate argument in which we prove the desired result for "good" partitions, while also characterizing the "bad" partitions where we do not get finiteness and showing that these bad partitions contradict the assumption that the matrix $A$ has super-rank $r$.

\section{Related work on bounding the number of exceptional subvarieties in diophantine problems}

In this section we briefly mention some earlier work in which authors have used $S$-unit equation bounds to show that most Diophantine problems of various types have at most the generically expected number of solutions.

We start with work of Evertse et al. [6] in which they show that up to equivalence, there are only finitely many equations of the form $a x+b y=c$ that have three or more solutions in $S$-units $x, y \in R_{S}^{*}$. We sketch their proof. Assuming that there are three solutions, one can eliminate $a / c$ and $b / c$ to obtain the determinantal equation

$$
0=\operatorname{det}\left(\begin{array}{lll}
x_{1} & y_{1} & 1 \\
x_{2} & y_{2} & 1 \\
x_{3} & y_{3} & 1
\end{array}\right)=x_{1} y_{2}-x_{2} y_{1}-x_{1} y_{3}+x_{3} y_{1}+x_{2} y_{3}-x_{3} y_{2} .
$$

This gives a six-term $S$-unit equation. If no subsum vanishes, they're essentially done. To finish the proof, they do a case-by-case analysis of five special cases where various subsums vanish. This proof has some features in common with our proof of Theorem 1.3, but our determinants are of arbitrary size, so the "case-by-case" analysis must cover all possible ways in which subsums of a multi-term sum can vanish.

In the higher dimensional case, Evertse [5] considers a finitely generated subgroup $\Gamma$ of $K^{n}$. He proves that there is a finite union $\mathcal{A}$ of $\Gamma$-equivalence classes of $n$-tuples $\left(a_{1}, \ldots, a_{n}\right)$ such that for all $n$-tuples not in $\mathcal{A}$, the set of non-degenerate solutions to the equation

$$
a_{1} x_{1}+\cdots+a_{n} x_{n}=1, \quad x_{1}, \ldots, x_{n} \in \Gamma,
$$

lies in the union of $2^{n}$ proper linear subspaces of $K^{n}$. His proof relies on a result of Laurent [15] for the number of non-degenerate points in $X \cap \Gamma$, where $X$ is an algebraic subvariety of $\mathbb{G}_{m}^{n}$. Rémond [16] has generalized Evertse's result to semiabelian varieties.

Perhaps closest to the present work is a paper of Schlickewei and Viola [17]. For fixed $\alpha_{1}, \ldots, \alpha_{n} \in K^{*}$ such that no ratio $\alpha_{i} / \alpha_{j}$ is a root of unity, they consider solutions to the determinantal equation

$$
F\left(y_{2}, \ldots, y_{n}\right)=\operatorname{det}\left(\begin{array}{cccc}
1 & 1 & \cdots & 1 \\
\alpha_{1}^{y_{2}} & \alpha_{2}^{y_{2}} & \cdots & \alpha_{n}^{y_{2}} \\
\vdots & & \cdots & \vdots \\
\alpha_{1}^{y_{n}} & \alpha_{2}^{y_{n}} & \cdots & \alpha_{n}^{y_{n}}
\end{array}\right)=0, \quad y_{2}, \ldots, y_{n} \in \mathbb{Z} .
$$

They prove that equation $(2.1)$ has at most $\left.\exp \left((6 n !)^{3 n !}\right)\right)$ solutions with the property that all proper subdeterminants of the matrix appearing in (2.1) are non-zero. They conjecture that a similar result is true under the weaker assumption that every $(n-1)$ by- $n$ and every $n$-by- $(n-1)$ submatrix has rank $n-1$. 
The theorem and conjecture of Schlickewei and Viola are both stronger and weaker than our main results. Our results are weaker in two ways. First, we assume that $\alpha_{1}, \ldots, \alpha_{n}$ are multiplicatively independent. Second, we essentially end up considering equations of the form

$$
F\left(d^{z_{2}}, \ldots, d^{z_{n}}\right)=0
$$

where $d \geq 2$ is a fixed integer, so we require that Schlickewei and Viola's variables $y_{2}, \ldots, y_{n}$ be powers of $d$.

Our results are also stronger in two ways. First, we only require that every $(n-1)$ by- $n$ submatrix of (2.1) have rank $n-1$, which is less stringent than even their conjectural requirement. Second, we work much more generally with an $r$-by- $n$ matrix, with the assumption that it has rank $r-1$ and that each of its $(r-1)$-by- $n$ submatrices also has rank $r-1$. It would be interesting to see if Schlickewei and Viola's result is true in this $r$-by- $n$ setting, subject of course to their strong assumption that every subdeterminant is non-zero.

Both our proof and the proof of Schlickewei and Viola use in a fundamental way the theorem of Evertse et al. [7] bounding the number of solutions to linear equations taking values in a finitely generated group. The proofs resemble one another in that they require intricate manipulations of the various ways in which subsums of a determinantal sum can vanish, but the proofs differ in many details due to their differing assumptions.

\section{Exceptional linear subspaces}

For the remainder of this paper we fix an algebraically closed field $K$ of characteristic 0 . Unless we indicate otherwise, all varieties, maps, and points are assumed to be defined over $K$. We also note that we use square brackets to denote homogeneous coordinates. Throughout $r$ denotes an integer in $\{1, \ldots, n\}$.

Definition 3.1. Let $L \subsetneq \mathbb{P}^{n}$ be a linear space of dimension $r-1$, so $r$ points in general position on $L$ will span $L$. A set of $r+1$ points

$$
S=\left\{Q_{0}, Q_{1}, \ldots, Q_{r}\right\} \subset L
$$

is said to super-span $L$ if every subset of $S$ containing $r$ points spans $L$.

Similarly, an $(r+1)$-by- $(n+1)$ matrix $A$ is said to have super-rank $r$ if $A$ has rank $r$ and further every submatrix consisting of $r$ rows of $A$ has rank $r$. From these definitions, if we let $S$ denote the set of points in $\mathbb{P}^{n}$ corresponding to the rows of $A$, then $A$ has super-rank $r$ if and only if $S$ super-spans an $(r-1)$-dimensional linear subspace of $\mathbb{P}^{n}$.

Definition 3.2. Let $\phi: \mathbb{P}^{n} \rightarrow \mathbb{P}^{n}$ be a morphism, and let $P \in \mathbb{P}^{n}$ be a point. For $r \geq 1$ we define the set of exceptional linear spaces for $\phi$ and $P$ to be the set

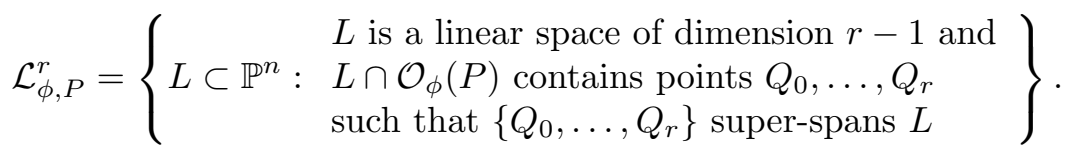

Using this notation, we can rewrite Conjecture 1.2 as follows. 
Conjecture 3.3. Let $\phi: \mathbb{P}^{n} \rightarrow \mathbb{P}^{n}$ be a morphism of degree $d \geq 2$, and let $P \in \mathbb{P}^{n}$ be a point whose orbit $\mathcal{O}_{\phi}(P)$ is Zariski dense in $\mathbb{P}^{n}$. Then for all $r \geq 1$, the set of exceptional linear spaces $\mathcal{L}_{\phi, P}^{r}$ is finite, and $\# \mathcal{L}_{\phi, P}^{r}$ may be bounded solely in terms of $n$ and $d$.

Remark 3.4. For a given map, it is easy to find initial points that lead to at least a few exceptional linear spaces. Thus fix a morphism $\phi: \mathbb{P}^{n} \rightarrow \mathbb{P}^{n}$, and let $0<m_{0}<$ $m_{1}<\cdots<m_{r}$ be a list of integers. Treating the coordinates of $P=\left[\alpha_{0}, \ldots, \alpha_{n}\right]$ as indeterminates, the condition that $\phi^{m_{0}}(P), \phi^{m_{1}}(P), \ldots, \phi^{m_{r}}(P)$ span a linear space of dimension $r-1$ is equivalent to requiring that $n-r+1$ determinants vanish, so it puts $n-r+1$ constraints on the coordinates of $P$. The super-spanning condition is Zariski open, so we will ignore it. If we choose another list of $r+1$ iterates to lie in a linear space of dimension $r-1$, we get another $n-r+1$ conditions on $P$. Hence, generically it should be possible to choose $P$ so that

$$
\# \mathcal{L}_{\phi, P}^{r} \geq\left\lfloor\frac{n}{n-r+1}\right\rfloor \text {. }
$$

(Note that the coordinates of $P$ and the linearity conditions are homogeneous.) So for example, for most $\phi: \mathbb{P}^{n} \rightarrow \mathbb{P}^{n}$ one expects that there exist initial points $P$, such that the orbit of $P$ super-spans $n$ distinct hyperplanes, which is the case $r=n$. This also suggests that any effective bound for

$$
\max \left\{\begin{array}{c}
\text { there exist } 0<m_{0}<\cdots<m_{r}=M \text { such } \\
M: \text { that } \phi^{m_{0}}(P), \ldots, \phi^{m_{r}}(P) \text { superspan } \\
\text { a linear subspace of dimension } r-1
\end{array}\right\}
$$

must depend on $P$, since in our construction we can take $m_{r}$ to be arbitrarily large.

Example 3.5. We illustrate Remark 3.4 using the map

$$
\phi: \mathbb{P}^{2} \longrightarrow \mathbb{P}^{2}, \quad \phi([x, y, z])=\left[x^{2}, y^{2}, z^{2}\right] .
$$

We will find an initial point $P=[\alpha, \beta, \gamma]$ so that $\# \mathcal{L}_{\phi, P}^{1} \geq 2$, i.e., so that $\mathcal{O}_{\phi}(P)$ super-spans at least two lines. The condition that $P, \phi(P)$, and $\phi^{2}(P)$ be colinear, i.e., they super-span a line, is

$$
\operatorname{det}\left(\begin{array}{ccc}
\alpha & \beta & \gamma \\
\alpha^{2} & \beta^{2} & \gamma^{2} \\
\alpha^{4} & \beta^{4} & \gamma^{4}
\end{array}\right)=\alpha \beta \gamma(\alpha-\beta)(\beta-\gamma)(\gamma-\alpha)(\alpha+\beta+\gamma)=0
$$

Similarly, the condition that $P, \phi^{3}(P)$, and $\phi^{4}(P)$ are colinear is

$$
\operatorname{det}\left(\begin{array}{ccc}
\alpha & \beta & \gamma \\
\alpha^{8} & \beta^{8} & \gamma^{8} \\
\alpha^{16} & \beta^{16} & \gamma^{16}
\end{array}\right)=\alpha \beta \gamma(\alpha-\beta)(\beta-\gamma)(\gamma-\alpha) h(\alpha, \beta, \gamma)=0,
$$

where $h$ is a complicated homogeneous polynomial of degree 19. Hence, $\phi$ and $P$ will have two exceptional lines in $\mathbb{P}^{2}$, i.e., $\# \mathcal{L}_{\phi, P}^{2} \geq 2$, if $P$ is chosen to satisfy the two simultaneous equations (3.1) and (3.2) with $\alpha \beta \gamma \neq 0$ and $\alpha, \beta, \gamma$ distinct. Dehomogenizing $\gamma=1$ and discarding solutions in which $\alpha$ or $\beta$ is a root of unity, we find that $P$ has the form $P=[\alpha, \beta, 1]$ with $\alpha$ and $\beta$ roots of the polynomial

$$
2 x^{6}+6 x^{5}+5 x^{4}+5 x^{2}+6 x+2 \text {, }
$$


such that $\alpha+\beta=-1$. The roots of this polynomial have the form

$$
\left\{x_{1}, \bar{x}_{1}, x_{1}^{-1}, \bar{x}_{1}^{-1}, x_{2}, \bar{x}_{2}\right\} \quad \text { with } \quad x_{1}+x_{2}=x_{1}^{-1}+\bar{x}_{1}^{-1}=-1 .
$$

Since we want $\alpha$ and $\beta$ to be multiplicatively independent, it suffices to take $\alpha=$ $x_{1} \approx-1.6243-0.7812 i$ and $\beta=x_{2} \approx 0.6243+0.7812 i$. Since $|\alpha|>1$ and $|\beta|=1$, it is clear that they are multiplicatively independent.

\section{Finiteness of exceptional linear subspaces for the $d$-power map}

Our main result says that subject to a multiplicative independence assumption on the coordinates of the point $P$, a strengthened form of the finiteness conjecture (Conjecture 3.3) is true for the $d^{\text {th }}$-power map on $\mathbb{P}^{n}$.

Definition 4.1. Let $P=\left[\alpha_{0}, \alpha_{1}, \ldots, \alpha_{n}\right] \in \mathbb{P}^{n}$ be a point not contained in any coordinate hyperplane, i.e., satisfying $\alpha_{0} \alpha_{1} \cdots \alpha_{n} \neq 0$. We define the multiplicative relation set of $P$ to be the set

$$
\mathcal{R}(P)=\left\{\mathbf{e}=\left(e_{0}, \ldots, e_{n}\right) \in \mathbb{Z}^{n+1}: \sum_{i=0}^{n} e_{i}=0 \text { and } \prod_{i=0}^{n} \alpha_{i}^{e_{i}}=1\right\} .
$$

We observe that $\mathcal{R}(P)$ is a sublattice of $\mathbb{Z}^{n+1}$.

Theorem 4.2. Let $\phi: \mathbb{P}^{n} \rightarrow \mathbb{P}^{n}$ be the $d^{\text {th }}$-power map

$$
\phi\left(\left[z_{0}, \ldots, z_{n}\right]\right)=\left[z_{0}^{d}, \ldots, z_{n}^{d}\right] .
$$

Fix a point $P \in \mathbb{P}^{n}$ whose relation set satisfies $\mathcal{R}(P)=\{\mathbf{0}\}$. Then for all $0 \leq r \leq n$, the set of exceptional linear spaces $\mathcal{L}_{\phi, P}^{r}$ is finite. Furthermore, there is an upper bound for $\# \mathcal{L}_{\phi, P}^{r}$ that depends only on $n$, independent of the point $P$ and the degree $d$.

Remark 4.3. Theorem 4.2 deals with linear subspaces of arbitrary dimension, but on first reading it may be easier for the reader to consider the case of hyperplanes, i.e., $r=n$. In particular, setting $r=n$ means that the set $\mathcal{P}_{r, n}$ defined after the proof of Lemma 4.4 contains only one element, namely the identity map on $\{0, \ldots, n\}$, which significantly simplifies the exposition.

Proof. First, we observe that if any coordinate of $P$ is 0 , then we can discard that coordinate and work on a lower dimensional projective space. So by induction on $n$, we may assume that $P$ does not lie in any of the coordinate hyperplanes.

Second, we note that the assumption that $\mathcal{R}(P)=\{\mathbf{0}\}$ implies that $P$ is not preperiodic, since $\phi^{m}(P)=P$ implies that $\alpha_{i}^{d^{m}-1} \alpha_{j}^{1-d^{m}}=1$ for all $i$ and $j$. (Of course, if $P$ is preperiodic, it is obvious that $\mathcal{L}_{\phi, P}^{r}$ is finite, since a finite set of points (super)spans only finitely many linear spaces. But it is not clear that there is a uniform bound for $\# \mathcal{L}_{\phi, P}^{r}$ independent of $P$.)

For the initial part of our proof, it suffices to assume that $P$ is not preperiodic and does not lie in a coordinate hyperplane. For possible future applications, we start with only these assumptions, and we will indicate where our proof first uses the stronger condition $\mathcal{R}(P)=\{\mathbf{0}\}$.

Write

$$
P=\left[\alpha_{0}, \alpha_{1}, \ldots, \alpha_{n}\right]
$$


where by assumption we have $\alpha_{0} \alpha_{1} \cdots \alpha_{n} \neq 0$. For any $(r+1)$-tuple of integers $\mathbf{m}=$ $\left(m_{0}, \ldots, m_{r}\right)$, we define an $(r+1)$-by- $(n+1)$ matrix (depending on $P=\left[\alpha_{0}, \ldots, \alpha_{n}\right]$ )

$$
A_{\mathbf{m}}=\left(\alpha_{j}^{d^{m_{i}}}\right)_{\substack{0 \leq i \leq r \\ 0 \leq j \leq n}} .
$$

In other words, the $i^{\text {th }}$ row of $A_{\mathbf{m}}$, considered as a point in $\mathbb{P}^{n}$, is the point $\phi^{m_{i}}(P)$. We then define a collection of exceptional $r+1$-tuples of iterates by

$$
\mathcal{M}_{P}=\left\{\mathbf{m} \in \mathbb{Z}^{r+1}: 0 \leq m_{0}<\cdots<m_{r} \text { and super-rank } A_{\mathbf{m}}=r\right\} .
$$

We also define

$$
L_{\mathbf{m}}=\left(\begin{array}{l}
\text { the linear subspace of } \mathbb{P}^{n} \text { spanned by the } \\
\text { points whose homogeneous coordinates } \\
\text { are the row vectors of the matrix } A_{\mathbf{m}}
\end{array}\right) .
$$

For $\mathbf{m} \in \mathcal{M}_{P}$, the rank condition on $A_{\mathbf{m}}$ implies that $L_{\mathbf{m}}$ is a linear subspace of exact dimension $r-1$, and the super-rank condition says that $L_{\mathbf{m}}$ is spanned by any $r$ of the $r+1$ rows of $A_{\mathbf{m}}$.

Lemma 4.4. The map

$$
\mathcal{M}_{P} \longrightarrow \mathcal{L}_{\phi, P}^{r}, \quad \mathbf{m} \longrightarrow L_{\mathbf{m}}
$$

is surjective.

Proof. The fact that $L_{\mathbf{m}}$ lies in $\mathcal{L}_{\phi, P}^{r}$ follows directly from the definitions of $\mathcal{M}_{P}$ and $\mathcal{L}_{\phi, P}^{r}$. For the surjectivity, let $L \in \mathcal{L}_{\phi, P}^{r}$. Then $L$ contains $r+1$ points in the orbit $\mathcal{O}_{\phi}(P)$, which we can label as $\phi^{m_{0}}(P), \ldots, \phi^{m_{r}}(P)$ with $0 \leq m_{0}<m_{1}<\cdots<$ $m_{r}$. We set $\mathbf{m}=\left(m_{0}, \ldots, m_{r}\right)$. Since

$$
\phi^{m_{j}}(P)=\left[\alpha_{0}^{d^{m_{j}}}, \alpha_{1}^{d^{m_{j}}}, \cdots, \alpha_{n}^{d^{m_{j}}}\right],
$$

the definition of $\mathcal{L}_{\phi, P}^{r}$ implies that the matrix $A_{\mathbf{m}}$ has super-rank $n$, so $\mathbf{m}$ is in $\mathcal{M}_{P}$, and by construction, the image of $\mathbf{m}$ in $\mathcal{L}_{\phi, P}^{r}$ is $L$.

Remark 4.5. The map (4.2) in Lemma 4.4 need not be injective. For example, if there is a linear space $L \in \mathcal{L}_{\phi, P}^{r}$ such that $L \cap \mathcal{O}_{\phi}(P)$ contains $r+2$ points such that every subset consisting of $r$ points spans $L$, then the cardinality of the preimage of $L$ is at least $\left(\begin{array}{c}r+2 \\ r+1\end{array}\right)=r+2$.

Lemma 4.4 reduces the proof of Theorem 4.2 to the following statement, whose proof occupies the remainder of this section.

Theorem 4.6. The set $\mathcal{M}_{P}$ is finite, and there is an upper bound for $\# \mathcal{M}_{P}$ that depends only on $n$, independent of the point $P$ and the degree $d$.

Proof. We define some additional notation. First, in order to select $r+1$ columns of a matrix $A_{\mathbf{m}}$, we look at the set of maps

$$
\mathcal{P}_{r, n}=\left\{\begin{array}{l}
\text { strictly increasing maps } \\
p:\{0, \ldots, r\} \rightarrow\{0, \ldots, n\}
\end{array}\right\} .
$$

For example, if $r=n$, then $p$ is necessarily the identity map, while if $r=n-1$, then there are exactly $n+1$ maps $p$, each of which is determined by the one value between 0 
and $n$ that is not in the image of $p$. For each $p \in \mathcal{P}_{r, n}$, we define an $(r+1)$-by- $(r+1)$ submatrix of $A_{\mathbf{m}}$ by

$$
A_{\mathbf{m}, p}=\left(\alpha_{p(j)}^{d^{m_{i}}}\right)_{\substack{0 \leq i \leq r \\ 0 \leq j \leq r}}
$$

In other words, the $(r+1)$-by- $(r+1)$ matrix $A_{\mathbf{m}, p}$ is obtained from the $(r+1)$-by- $(n+1)$ matrix $A_{\mathbf{m}}$ by taking columns $p(0), p(1), \ldots, p(r)$.

To ease notation, we also let

$$
\begin{aligned}
k_{i}(\mathbf{m}) & =d^{m_{i}} & & \text { for } 0 \leq i \leq r, \\
u_{\sigma, p}(\mathbf{m}) & =\alpha_{p(0)}^{k_{\sigma(0)}(\mathbf{m})} \alpha_{p(1)}^{k_{\sigma(1)}(\mathbf{m})} \cdots \alpha_{p(r)}^{k_{\sigma(r)}(\mathbf{m})} & & \text { for } \sigma \in \mathcal{S}_{r+1} \text { and } p \in \mathcal{P}_{r, n} .
\end{aligned}
$$

With this notation, the determinant of the matrix $A_{\mathbf{m}, p}$ is

$$
\operatorname{det}\left(A_{\mathbf{m}, p}\right)=\operatorname{det}\left(\alpha_{p(j)}^{k_{i}(\mathbf{m})}\right)_{\substack{0 \leq i \leq r \\ 0 \leq j \leq r}}=\sum_{\sigma \in \mathcal{S}_{r+1}} \operatorname{sgn}(\sigma) u_{\sigma, p}(\mathbf{m}) .
$$

To simplify notation, we will often suppress the dependence on $\mathbf{m}$ and just write $k_{i}$ and $u_{\sigma, p}$, but we stress that our eventual goal is to show that there are only finitely many $\mathbf{m}$ satisfying certain conditions, so most of our formulas should be viewed as relations on the unknown quantity $\mathbf{m}$.

The following elementary lemma will be useful.

Lemma 4.7. Let $d \geq 2$ be an integer, and let $a, b, x, y$ be non-negative integers. Suppose that

$$
d^{a}-d^{b}=d^{x}-d^{y} .
$$

Then $\{a, y\}=\{b, x\}$, i.e., either

$$
(a=x \text { and } b=y) \quad \text { or }(a=b \text { and } x=y) \text {. }
$$

Proof. Multiplying the relation (4.3) by -1 if necessary, we may assume that $a \geq b$, and then since the left-hand side is positive, we also have $x \geq y$. From (4.3) it is clear that if $a=b$, then $x=y$, and similarly, if $x=y$, then $a=b$. We are thus reduced to the case that $a>b$ and $x>y$. Factoring (4.3) gives

$$
d^{b}\left(d^{a-b}-1\right)=d^{y}\left(d^{x-y}-1\right) .
$$

Since $d^{a-b}-1$ and $d^{x-y}-1$ are both relative prime to $d$ (this is where we use the assumption that $a>b$ and $x>y$ ), we find that $d^{b}=d^{y}$, so $b=y$. It is then clear from (4.3) that also $a=x$.

We next use Lemma 4.7 to show that the values of the products $u_{\sigma, p}(\mathbf{m})$ determine the value of $\mathbf{m}$.

Lemma 4.8. Let $p \in \mathcal{P}_{r, n}$ have the property that the point

$$
\left[\alpha_{p(0)}, \alpha_{p(1)}, \ldots, \alpha_{p(r)}\right]
$$

is not preperiodic for the $d^{\text {th }}$-power map, i.e., at least one of the ratios $\alpha_{p(j)} / \alpha_{p(0)}$ is not a root of unity. Then the map

$$
\mathcal{M}_{P} \longrightarrow \mathbb{P}^{(r+1) !-1}, \quad \mathbf{m} \longrightarrow\left[u_{\sigma, p}(\mathbf{m})\right]_{\sigma \in \mathcal{S}_{r+1}},
$$

is injective. 
Proof. Suppose that $\mathbf{m}$ and $\widetilde{\mathbf{m}}$ are elements of $\mathcal{M}_{P}$ that have the same image in $\mathbb{P}^{(r+1) !-1}$. This means that

$$
\prod_{i} \alpha_{p(i)}^{k_{\sigma(i)}} \alpha_{p(i)}^{\widetilde{k}_{\tau(i)}}=\prod_{i} \alpha_{p(i)}^{k_{\tau(i)}} \alpha_{p(i)}^{\widetilde{k}_{\sigma(i)}}, \quad \text { for all } \sigma, \tau \in S_{r+1}
$$

Applying (4.5) with $\sigma=\mathrm{id}$ and $\tau$ the transposition $\tau=(0 j)$ gives the relation

$$
\left(\alpha_{p(j)} / \alpha_{p(0)}\right)^{k_{j}-\widetilde{k}_{j}-k_{0}+\widetilde{k}_{0}}=1 .
$$

This holds for all $0 \leq j \leq n$. We are assuming that at least one of the ratios $\alpha_{p(j)} / \alpha_{p(0)}$ is not a root of unity, say $\alpha_{p(t)} / \alpha_{p(0)}$ is not a root of unity. Then the exponent on the left-hand side of (4.6) must vanish, so we find that

$$
k_{t}-k_{0}=\tilde{k}_{t}-\tilde{k}_{0} .
$$

Using the definition of $k_{j}=k_{j}(\mathbf{m})$, this is equivalent to the equation

$$
d^{m_{t}}-d^{m_{0}}=d^{\widetilde{m}_{t}}-d^{\widetilde{m}_{0}} .
$$

The fact that $\mathbf{m} \in \mathcal{M}_{P}$ means that $m_{t}>m_{0}$, so (4.7) and Lemma 4.7 imply that

$$
m_{0}=\widetilde{m}_{0} \quad \text { and } \quad m_{t}=\widetilde{m}_{t} .
$$

We now take an arbitrary index $i$ and consider the multiplicative relation (4.5) for the permutations $\sigma=(0 i t)$ and $\tau=(0 t i)$. After canceling common terms from the two sides of the equation, we are left with the formula

$$
\alpha_{p(0)}^{k_{i}} \alpha_{p(i)}^{k_{t}} \alpha_{p(t)}^{k_{0}} \alpha_{p(0)}^{\tilde{k}_{t}} \alpha_{p(t)}^{\tilde{k}_{i}} \alpha_{p(i)}^{\tilde{k}_{0}}=\alpha_{p(0)}^{\tilde{k}_{i}} \alpha_{p(i)}^{\tilde{k}_{t}} \alpha_{p(t)}^{\tilde{k}_{0}} \alpha_{p(0)}^{k_{t}} \alpha_{p(t)}^{k_{i}} \alpha_{p(i)}^{k_{0}}
$$

However, we already know from (4.8) that $k_{0}=\tilde{k}_{0}$ and $k_{t}=\tilde{k}_{t}$. This allows us to cancel many of the terms in (4.9), and we find that

$$
\left(\alpha_{p(0)} / \alpha_{p(t)}\right)^{k_{i}-\tilde{k}_{i}}=1
$$

We know that $\alpha_{p(0)} / \alpha_{p(t)}$ is not a root of unity, since that's how we chose $t$, so it follows that $k_{i}=\tilde{k}_{i}$. This is true for all $i$, and since $k_{i}=d^{m_{i}}$ and $\tilde{k}_{i}=d^{\tilde{m}_{i}}$, we have proven that $m_{i}=\tilde{m}_{i}$ for all $0 \leq i \leq r$. Hence $\mathbf{m}=\tilde{\mathbf{m}}$, which completes the proof that the map (4.4) is injective.

We resume the proof of Theorem 4.6. Let $\mathbf{m} \in \mathcal{M}_{P}$. Then the $(r+1)$-by- $(n+1)$ matrix $A_{\mathbf{m}}$ has rank $r$, so all of its $(r+1)$-by- $(r+1)$ minors vanish. In our notation,

$$
\operatorname{det} A_{\mathbf{m}, p}=\sum_{\sigma \in \mathcal{S}_{r+1}} \operatorname{sgn}(\sigma) u_{\sigma, p}(\mathbf{m})=0 \quad \text { for all } p \in \mathcal{P}_{r, n} .
$$

We will use the following deep result on $S$-unit equations, which we will apply with

$$
\Gamma=\left\{\text { subgroup of } K^{*} \text { generated by }-1, \alpha_{0}, \ldots, \alpha_{n}\right\} .
$$

Theorem 4.9. Let $K$ be a field of characteristic 0 , let $\Gamma$ be a finitely generated subgroup of $K^{*}$, and let $a_{0}, \ldots, a_{N} \in \Gamma$. Then the equation

$$
a_{0} u_{0}+a_{1} u_{1}+\cdots+a_{N} u_{N}=0
$$


has only finitely many solutions $\left[u_{0}, \ldots, u_{N}\right] \in \mathbb{P}^{N}(K)$ satisfying

$$
u_{0}, \ldots, u_{N} \in \Gamma
$$

and

$$
\sum_{i \in I} a_{i} u_{i} \neq 0 \quad \text { for all non-empty subsets } I \subsetneq\{0,1, \ldots, N\} .
$$

Further, there is a bound for the number of such solutions that depends solely on $N$ and $\operatorname{rank}(\Gamma)$.

Proof. See [1, Theorem 6.2] or [7] for explicit upper bounds.

As a warm-up, we first consider the set of elements $\mathbf{m} \in \mathcal{M}_{P}$ such that for every $p \in$ $\mathcal{P}_{r, n}$, no subsum in the determinant equation (4.10) equals 0 . Theorem 4.9 tells us that the equation

$$
\sum_{\sigma \in \mathcal{S}_{r+1}} \operatorname{sgn}(\sigma) v_{\sigma}=0
$$

has only finitely many solutions in $\mathbb{P}^{(r+1) !-1}(K)$ with $v_{\sigma} \in \Gamma$ and such that no subsum equals 0 . Hence, there are only finitely many possible values for the point

$$
\left[u_{\sigma, p}(\mathbf{m})\right]_{\sigma \in \mathcal{S}_{r+1}} \in \mathbb{P}^{(r+1) !-1}(K) .
$$

The assumption that $P$ is not preperiodic means that at least one ratio $\alpha_{t} / \alpha_{0}$ is not a root of unity. We take $p \in \mathcal{P}_{r, n}$ such that 0 and $t$ are in the image of $p$, which allows us to apply Lemma 4.8 to conclude that the map (4.4) is injective. Hence, there are only finitely many values for $\mathbf{m}$. Furthermore, the uniformity in Theorem 4.9 gives a uniform upper bound for $\mathcal{M}_{P}$.

We now consider the general case in which one or more subsums in (4.10) may be equal to 0 . In this case the conclusion of Theorem 4.9 is false, since we can scale individual zero subsums. In general, we look at partitions $\mathcal{I}$ of $\mathcal{S}_{r+1}$, i.e.,

$$
\mathcal{I}=\left\{T_{1}, \ldots, T_{s}\right\} \quad \text { with } \quad T_{i} \cap T_{j}=\emptyset \quad \text { and } \quad T_{1} \cup \cdots \cup T_{s}=\mathcal{S}_{r+1} .
$$

For each $p \in \mathcal{P}_{r, n}$ we want to choose a maximal partition $\mathcal{I}_{p}$ of $\mathcal{S}_{r+1}$ such that

$$
\sum_{\sigma \in T} \operatorname{sgn}(\sigma) u_{\sigma, p}(\mathbf{m})=0 \quad \text { for all } T \in \mathcal{I}_{p} .
$$

Let

$$
\mathcal{V}=\left\{\left[v_{\sigma}\right]_{\sigma \in \mathcal{S}_{r+1}} \in \mathbb{P}^{(r+1) !-1}: v_{\sigma} \in \Gamma \text { and } \sum_{\sigma \in \mathcal{S}_{r+1}} \operatorname{sgn}(\sigma) v_{\sigma}=0\right\},
$$

and for each partition $\mathcal{I}$ of $\mathcal{S}_{r+1}$, define

$$
\mathcal{V}_{\mathcal{I}}=\left\{\left[v_{\sigma}\right] \in \mathcal{V}: \begin{array}{c}
\sum_{\sigma \in T} \operatorname{sgn}(\sigma) v_{\sigma}=0 \text { for all } T \in \mathcal{I}, \text { and } \\
\sum_{\sigma \in T^{\prime}} \operatorname{sgn}(\sigma) v_{\sigma} \neq 0 \text { for all } T^{\prime} \subsetneq T \in \mathcal{I}
\end{array}\right\} .
$$


We note that

$$
\mathcal{V}=\bigcup_{\substack{\mathcal{I} \text { is a partition } \\ \text { of } \mathcal{S}_{n+1}}} \mathcal{V}_{\mathcal{I}}
$$

although the $\mathcal{V}_{\mathcal{I}}$ are not necessarily disjoint.

For each $p \in \mathcal{P}_{r, n}$ we now fix a partition $\mathcal{I}_{p}$ of $\mathcal{S}_{n+1}$. This choice of partitions will be fixed for the remainder of the proof. We claim that the set

$$
\left\{\left(\left[u_{\sigma, p}(\mathbf{m})\right]_{\sigma \in \mathcal{S}_{r+1}}\right)_{p \in \mathcal{P}_{r, n}}: \mathbf{m} \in \mathcal{M}_{P}\right\} \cap \prod_{p \in \mathcal{P}_{r, n}} \mathcal{V}_{\mathcal{I}_{p}}
$$

is finite (and has order bounded in terms of $n$ ). This claim will complete the proof of Theorem 4.6, since from the definitions it is clear that for all $p$,

$$
\left\{\left[u_{\sigma, p}(\mathbf{m})\right]_{\sigma \in \mathcal{S}_{r+1}}: \mathbf{m} \in \mathcal{M}_{P}\right\} \subset \mathcal{V}
$$

and Lemma 4.8 tells us that the value of $\left(\left[u_{\sigma, p}(\mathbf{m})\right]_{\sigma \in \mathcal{S}_{r+1}}\right)_{p \in \mathcal{P}_{r, n}}$ determines the value of $\mathbf{m}$.

The definition of $\mathcal{V}_{\mathcal{I}}$ says that for each $T \in \mathcal{I}$, a certain sum of $S$-units is zero and no subsum is zero. Hence we can apply Theorem 4.9 to each sum over $T$. In other words, if we map

$$
\mathcal{V}_{\mathcal{I}} \longrightarrow \prod_{T \in \mathcal{I}} \mathbb{P}^{\# T-1}, \quad\left[v_{\sigma}\right]_{\sigma \in \mathcal{S}_{n+1}} \longmapsto\left(\left[v_{\sigma}\right]_{\sigma \in T}\right)_{T \in \mathcal{I}},
$$

then the image of this map is finite and has order bounded in terms of $n$.

Hence in order to prove that the set (4.11) is finite, it suffices to show that the map

$$
\begin{aligned}
& \boldsymbol{F}: \mathcal{M}_{P} \longrightarrow \prod_{p \in \mathcal{P}_{r, n}} \prod_{T \in \mathcal{I}_{p}} \mathbb{P}^{\# T-1}, \\
& \mathbf{m} \longmapsto\left(\left[u_{\sigma}(\mathbf{m})\right]_{\sigma \in T}\right)_{\substack{p \in \mathcal{P}_{r, n} \\
T \in \mathcal{I}_{p}}},
\end{aligned}
$$

is injective.

Lemma 4.10. For each $p \in \mathcal{P}_{r, n}$, let $\mathcal{J}_{p}$ be a subpartition of $\mathcal{I}_{p}$, i.e, for every $T \in \mathcal{J}_{p}$ there is a $T^{\prime} \in \mathcal{I}_{p}$ such that $T \subset T^{\prime}$. Write $F_{\mathcal{I}}$ for the map (4.12) using the $\mathcal{I}_{p}$ partitions, and write $F_{\mathcal{J}}$ for the map (4.12) using the $\mathcal{J}_{p}$ partitions. Then

$$
\boldsymbol{F}_{\mathcal{I}}(\mathbf{m})=\boldsymbol{F}_{\mathcal{I}}(\widetilde{\mathbf{m}}) \Longrightarrow \boldsymbol{F}_{\mathcal{J}}(\mathbf{m})=\boldsymbol{F}_{\mathcal{J}}(\widetilde{\mathbf{m}})
$$

Proof. Let $p \in \mathcal{P}_{r, n}$ and let $T \in \mathcal{J}_{p}$. Then there is a $T^{\prime} \in \mathcal{I}_{p}$ with $T \subset T^{\prime}$. The assumption that $\boldsymbol{F}_{\mathcal{I}}(\mathbf{m})=\boldsymbol{F}_{\mathcal{I}}(\widetilde{\mathbf{m}})$ means that here is a $\lambda \in K^{*}$ such that

$$
u_{\sigma, p}(\mathbf{m})=\lambda u_{\sigma, p}(\widetilde{\mathbf{m}}) \quad \text { for all } \sigma \in T^{\prime} .
$$

In particular, the equality (4.13) holds for all $\sigma \in T$, since $T \subset T^{\prime}$. Hence,

$$
\left[u_{\sigma}(\mathbf{m})\right]_{\sigma \in T}=\left[u_{\sigma}(\widetilde{\mathbf{m}})\right]_{\sigma \in T},
$$

and since this is true for all $T \in \mathcal{J}_{p}$, we conclude that $\boldsymbol{F}_{\mathcal{J}}(\mathbf{m})=\boldsymbol{F}_{\mathcal{J}}(\widetilde{\mathbf{m}})$. 
We now resume writing $\boldsymbol{F}$ for the map (4.12), i.e., we drop the $\mathcal{I}$ subscript. Suppose that

$$
\boldsymbol{F}(\mathbf{m})=\boldsymbol{F}(\widetilde{\mathbf{m}}) .
$$

This is equivalent to the statement that

$$
u_{\sigma, p}(\mathbf{m}) u_{\tau, p}(\widetilde{\mathbf{m}})=u_{\tau, p}(\mathbf{m}) u_{\sigma, p}(\widetilde{\mathbf{m}}) \quad \text { for all } p \in \mathcal{P}_{r, n} \text {, all } T \in \mathcal{I}_{p} \text {, and all } \sigma, \tau \in T \text {. }
$$

Replacing the $u_{\sigma, p}$ with their expressions as products of $\alpha_{i}$, this becomes

$$
\prod_{i=0}^{n} \alpha_{p(i)}^{k_{\sigma(i)}+\tilde{k}_{\tau(i)}-k_{\tau(i)}-\tilde{k}_{\sigma(i)}}=1 \quad \text { for all } p \in \mathcal{P}_{r, n}, \text { all } T \in \mathcal{I}_{p}, \text { and all } \sigma, \tau \in T .
$$

We now invoke the assumption that the relation set for the point $P$ is trivial, i.e.,

$$
\mathcal{R}(P)=\{\mathbf{0}\} \text {. }
$$

We note that

$$
\sum_{i=0}^{n}\left(k_{\sigma(i)}-k_{\tau(i)}\right)=\sum_{i=0}^{n}\left(\tilde{k}_{\tau(i)}-\tilde{k}_{\sigma(i)}\right)=0,
$$

so the assumption $\mathcal{R}(P)=\{\boldsymbol{0}\}$ implies that the exponents in (4.14) all vanish. Hence $k_{\sigma(i)}-k_{\tau(i)}=\tilde{k}_{\tau(i)}-\tilde{k}_{\sigma(i)} \quad$ for all $p \in \mathcal{P}_{r, n}$, all $T \in \mathcal{I}_{p}$, all $\sigma, \gamma \in T$, and all $0 \leq i \leq n$.

Rewriting using $k_{i}=k_{i}(\mathbf{m})=d^{m_{i}}$ yields

$$
\begin{aligned}
d^{m_{\sigma(i)}}-d^{m_{\tau(i)}}= & d^{\tilde{m}_{\sigma(i)}}-d^{\tilde{m}_{\tau(i)}} \\
& \text { for all } p \in \mathcal{P}_{r, n}, \text { all } T \in \mathcal{I}_{p}, \text { all } \sigma, \gamma \in T, \text { and all } 0 \leq i \leq n .
\end{aligned}
$$

Since the entries of $\mathbf{m}$ are distinct, as are the entries of $\widetilde{\mathbf{m}}$, we can use Lemma 4.7 to deduce that

$$
\sigma(i) \neq \tau(i) \Longrightarrow m_{\sigma(i)}=\widetilde{m}_{\sigma(i)} \quad \text { and } \quad m_{\tau(i)}=\widetilde{m}_{\tau(i)}
$$

This holds for all $0 \leq i \leq n$, all $p \in \mathcal{P}_{r, n}$, all $T \in \mathcal{I}_{p}$, and all $\sigma, \tau \in T$. It will be more convenient to use the contrapositive of (4.16). So suppose that $m_{t} \neq \widetilde{m}_{t}$ for some $t$. Taking $i=\sigma^{-1}(t)$ for some $\sigma \in T$, we deduce that

$$
m_{t} \neq \widetilde{m}_{t} \Longrightarrow \sigma\left(\sigma^{-1}(t)\right)=\tau\left(\sigma^{-1}(t)\right) .
$$

The conclusion may be rewritten as $\tau^{-1}(t)=\sigma^{-1}(t)$, so we have proven the following key implication:

$$
m_{t} \neq \tilde{m}_{t} \Longrightarrow \forall p \in \mathcal{P}_{r, n}, \quad \forall T \in \mathcal{I}_{p}, \quad \forall \sigma, \tau \in T, \quad \tau^{-1}(t)=\sigma^{-1}(t) .
$$

We would like to show that (4.17) gives enough relations to force $\mathbf{m}=\widetilde{\mathbf{m}}$, but we will need to use the super-spanning assumption, i.e., the assumption that $A_{\mathbf{m}}$ and $A_{\widetilde{\mathbf{m}}}$ have super-rank $r$, to eliminate some exceptional cases for which there are not enough relations.

Definition 4.11. For each $0 \leq t \leq r$ and each $0 \leq j \leq r$, we define sets

$$
T_{j}^{t}=\left\{\sigma \in \mathcal{S}_{r+1}: \sigma(j)=t\right\} .
$$

For each $t$, this gives a partition

$$
\mathcal{I}_{\bullet}^{t}=\left\{T_{0}^{t}, T_{1}^{t}, \ldots, T_{r}^{t}\right\}
$$


of $\mathcal{S}_{r+1}$. We will say that a partition of $\mathcal{S}_{r+1}$ is exceptional if it is a subpartition of $\mathcal{I}_{\bullet}^{t}$ for some $0 \leq t \leq r$. In particular, each partition $\mathcal{I}_{\bullet}^{t}$ is itself exceptional.

Lemma 4.12. Suppose that for some $p \in \mathcal{P}_{r, n}$, the partition $\mathcal{I}_{p}$ is not exceptional. Then the map $\boldsymbol{F}$ defined by (4.12) is injective.

More precisely, if $\boldsymbol{F}(\mathbf{m})=\boldsymbol{F}(\widetilde{\mathbf{m}})$ with $m_{t} \neq \widetilde{m}_{t}$, then every $\mathcal{I}_{p}$ is a subpartition of $\mathcal{I}_{\bullet}^{t}$.

Proof. We prove the second statement, so we assume that

$$
\boldsymbol{F}(\mathbf{m})=\boldsymbol{F}(\widetilde{\mathbf{m}}) \text { and } m_{t} \neq \widetilde{m}_{t},
$$

and we will prove that $\mathcal{I}_{p}$ is a subpartition of $\mathcal{I}_{\bullet}^{t}=\left\{T_{0}^{t}, \ldots, T_{n}^{t}\right\}$.

Let $T \in \mathcal{I}_{p}$ be any set in the partition, and let $\sigma, \tau \in T$. Applying (4.17), we conclude that

$$
\tau^{-1}(t)=\sigma^{-1}(t)
$$

Hence the set

$$
\left\{\sigma^{-1}(t): \sigma \in T\right\}
$$

contains only one number, which we denote by $j(T)$. In other words,

$$
\sigma(j(T))=t \quad \text { for all } \sigma \in T,
$$

so by definition of $T_{j}^{t}$, this means that $T \subset T_{j(T)}^{t}$, where we stress that the index $t$ does not depend on $p$ or $T$. Thus every $T \in \mathcal{I}_{p}$ is contained in one of the sets in the partition $\left\{T_{0}^{t}, \ldots, T_{n}^{t}\right\}$, so $\mathcal{I}_{p} \subset \mathcal{I}_{\text {. }}^{t}$. In particular, $\mathcal{I}_{p}$ is an exceptional partition, which completes the proof of Lemma 4.12 .

Remark 4.13. If any $\mathcal{I}_{p}$ is not exceptional, then Lemma 4.12 says that $\boldsymbol{F}$ is injective. We briefly indicate how exceptional partitions $\mathcal{I}_{p}$ can lead to $\boldsymbol{F}$ being non-injective. From Lemma 4.10, it suffices to look at the case that $\mathcal{I}_{p} \subset \mathcal{I}_{\bullet}^{t}$ for all $p$ and some $t$, since if these lead to non-injective maps $\boldsymbol{F}$, then so do their subpartitions.

For $\sigma \in T_{j}^{t}$, we compute

$$
u_{\sigma, p}(\mathbf{m})=\prod_{i=0}^{r} \alpha_{p(i)}^{k_{\sigma(i)}}=\alpha_{p(j)}^{k_{t}} \prod_{\substack{i=0 \\ i \neq j}}^{r} \alpha_{p(i)}^{k_{\sigma(i)}}=\alpha_{p(j)}^{k_{t}} \prod_{\substack{i=0 \\ i \neq t}}^{r} \alpha_{p\left(\sigma^{-1}(i)\right)}^{k_{i}},
$$

where the last equality uses that fact that $\sigma(j)=t$ for all $\sigma \in T_{j}^{t}$. The quantity $\alpha_{p(j)}^{k_{t}}$ does not depend on $\sigma$, so it may be canceled from homogeneous coordinates, yielding (where we recall that $k_{i}=d^{m_{i}}$ )

$$
\left[u_{p, \sigma}(\mathbf{m})\right]_{\sigma \in T_{j}^{t}}=\left[\prod_{\substack{i=0 \\ i \neq t}}^{n} \alpha_{p\left(\sigma^{-1}(i)\right)}^{m^{m_{i}}}\right]_{\sigma \in T_{j}^{t}} .
$$

Note that this formula for $\left[u_{p, \sigma}(\mathbf{m})\right]_{\sigma \in T_{j}^{t}}$ does not involve $m_{t}$. Hence $\boldsymbol{F}(\mathbf{m})$ does not depend on the $t^{\text {th }}$-coordinate of $\mathbf{m}$. Thus $\boldsymbol{F}$ is not injective if we consider it to be a map on the set of all integer vectors $\mathbf{m}=\left(m_{0}, \ldots, m_{r}\right)$. We will need to use the assumption that $\mathbf{m} \in \mathcal{M}_{P}$, i.e., that $A_{\mathbf{m}}$ has super-rank equal to $r$, to rule out with this potential non-injectivity. 
We resume the proof of Theorem 4.6, so in particular we assume that $\mathcal{R}(P)=\{\mathbf{0}\}$. Recall that for each $p \in \mathcal{P}_{r, n}$ we have fixed a partition $\mathcal{I}$ of $\mathcal{S}_{r+1}$ and used it in (4.12) to define a map $\boldsymbol{F}$. As indicated earlier, it suffices to prove that the set (4.11) is finite, and for this it suffices to prove that the map $\boldsymbol{F}$ is injective. Lemma 4.12 says that $\boldsymbol{F}$ is injective unless every $\mathcal{I}_{p}$ is exceptional for the same index $t$, i.e., unless there is an index $t$ such every $\mathcal{I}_{p}$ is contained in $\mathcal{I}_{\bullet}^{t}$. We deal with these exceptional cases in the following lemma, which says that in these cases, the set (4.11) is empty.

Lemma 4.14. Suppose that there is an index $t$ such that $\mathcal{I}_{p} \subset \mathcal{I}_{\bullet}^{t}$ for every $p \in \mathcal{P}_{r, n}$. Let $\mathbf{m}=\left(m_{0}, \ldots, m_{n}\right) \in \mathbb{Z}^{n+1}$ with $0 \leq m_{0}<m_{1}<\cdots<m_{n}$ have the property that

$$
\left(u_{\sigma, p}(\mathbf{m})\right)_{\sigma \in \mathcal{S}_{r+1}} \in \mathcal{V}_{\mathcal{I}_{p}} \quad \text { for all } p \in \mathcal{P}_{r, n} .
$$

Then $\mathbf{m} \notin \mathcal{M}_{P}$, i.e., the matrix $A_{\mathbf{m}}$ does not have super-rank equal to $r$. More precisely, if we delete the $t^{\text {th }}$ row of $A_{\mathbf{m}}$, the resulting matrix has rank $r-1$.

Proof. Assumption (4.20) and the definition of $\mathcal{V}_{\mathcal{I}_{p}}$ say that

$$
\sum_{\sigma \in T} \operatorname{sgn}(\sigma) u_{\sigma, p}(\mathbf{m})=0 \quad \text { for all } T \in \mathcal{I}_{p} .
$$

We are assuming that $\mathcal{I}_{p} \subset \mathcal{I}_{\bullet}^{t}$, so each $T^{\prime} \in \mathcal{I}_{\bullet}^{t}$ is a union of elements of $\mathcal{I}_{p}$. Summing (4.21) over the $T$ whose union is $T^{\prime}$, we find that (4.21) is true for the partition $\mathcal{I}_{\text {. }}^{t}$. Thus it suffices to prove the lemma under the assumption that $\mathcal{I}_{p}$ is equal to the maximal exceptional partition $\mathcal{I}_{\bullet}^{t}$.

As computed earlier, see (4.18), we have

$$
u_{\sigma}(\mathbf{m})=\alpha_{p(j)}^{k_{t}} \prod_{i \neq j} \alpha_{p(i)}^{k_{\sigma(i)}}, \quad \text { for all } \sigma \in T_{j}^{t}
$$

Hence in the sum (4.21) with $T=T_{j}^{t}$, we can cancel $\alpha_{p(j)}^{k_{t}}$ from every term, which yields

$$
\sum_{\sigma \in T_{j}^{t}} \operatorname{sgn}(\sigma) \prod_{i \neq j} \alpha_{p(i)}^{k_{\sigma(i)}}=0 .
$$

We observe that this sum is exactly the determinant of the matrix obtained by deleting the $t^{\text {th }}$ row and the $p(j)^{\text {th }}$ column from the matrix $A_{\mathbf{m}, p}$. (This is because the sum consists of the terms for which $i$ is never equal to $p(j)$ and $\sigma(i)$ is never equal to $t$.)

The value of $t$ is fixed, but $p$ and $j$ are arbitrary, so we have proven that if we delete the $t^{\text {th }}$ row of $A_{\mathbf{m}}$, then the resulting $r$-by- $(n+1)$ dimensional matrix has rank at most $r-1$, since all of its $r$-by- $r$ minors vanish. By the definition of superrank, it follows that $A_{\mathbf{m}}$ does not have super-rank $r$, which completes the proof of Lemma 4.14.

We now summarize how the results in this section fit together to prove Theorems 4.2 and 4.6.

(1) For each $p \in \mathcal{P}_{r, n}$, i.e., for each choice of an $(r+1)$-by- $(r+1)$ minor of the matrix $A_{\mathbf{m}}$, we fix a partition $\mathcal{I}_{p}$ of $\mathcal{S}_{r+1}$ that describes the minimal subsums of the determinant $\operatorname{det} A_{\mathbf{m}, p}$ that vanish. 
(2) The terms in the expansion of $\operatorname{det} A_{\mathbf{m}, p}$ lie in a finitely generated subgroup of $K^{*}$, so Theorem 4.9 says that there are only finitely many possibilities for the terms in each subsum, where the terms are viewed as a point in projective space.

(3) This implies that the image of the map $\boldsymbol{F}$ defined by (4.12) is finite, so to prove the required finiteness of $\mathcal{M}_{P}$, it suffices to show that $\boldsymbol{F}$ is injective for all choices of the partitions $\mathcal{I}_{p}$.

(4) Lemma 4.12 says that the map $\boldsymbol{F}$ is injective unless there is an index $t$ such that every $\mathcal{I}_{p}$ is a subpartition of the exceptional partition $\mathcal{I}_{\bullet}^{t}$. (A key point here is that one $t$ works for every $p$.)

(5) It remains to deal with the case that there is an index $t$ such that every $\mathcal{I}_{p}$ is a subpartition of $\mathcal{I}_{\bullet}^{t}$. But in this case, Lemma 4.14 says that the associated $(r+1)$-tuples $\mathbf{m}$ give matrices $A_{\mathbf{m}}$ that have rank $r-1$ when their $t^{\text {th }}$ rows are deleted. Thus $A_{\mathrm{m}}$ does not have super-rank $r$, so $\mathbf{m} \notin \mathcal{M}_{P}$.

(6) This shows that $\mathcal{M}_{P}$ is finite, which completes the proof of Theorem 4.6.

(7) Then Lemma 4.4, which says that $\mathcal{M}_{P}$ maps onto $\mathcal{L}_{\phi, P}^{r}$, which completes the proof of Theorem 4.2 .

\section{The size of the intersection $\mathcal{O}_{\phi}(P) \cap L$}

In this section, we give the proof of Corollary 1.4, which we restate for the convenience of the reader

Corollary 5.1. Let $\phi$ and $P$ be as in the statement of Theorem 1.3. Then for any linear subspace $L$, the intersection $\mathcal{O}_{\phi}(P) \cap L$ is finite, and its size is bounded solely in terms of $n$, independent of $P$ and $d$.

Proof. We consider anew the map $\mathcal{M}_{P} \longrightarrow \mathcal{L}_{\phi, P}^{r}$ defined in Lemma 4.4. Fix a linear space $L$ in the codomain, so

$$
\#\left(L \cap \mathcal{O}_{\phi}(P)\right) \geq r+1 .
$$

Then the preimage of $L$ under this map, i.e.,

$$
\mathcal{M}_{P}(L):=\left\{\mathbf{m} \in \mathcal{M}_{P}: L_{\mathbf{m}}=L\right\},
$$

consists of strictly increasing $(r+1)$-tuples $\mathbf{m}$ such that

$$
\left\{\phi^{m_{0}}(P), \ldots, \phi^{m_{r}}(P)\right\} \subseteq L \cap \mathcal{O}_{\phi}(P) .
$$

Thus a bound on $\# \mathcal{M}_{P}$ solely in term of $n$ gives an analogous bound on $\#\left(L \cap \mathcal{O}_{\phi}(P)\right)$ for any linear space $L$. Using this fact, Corollary 1.4 follows from Theorem 4.6.

\section{Orbits that are not Zariski dense}

It is interesting to ask if we can weaken the hypotheses of Conjecture 1.2. This is already a non-trivial question when $\phi$ is the $d^{\text {th }}$-power map, in which case we ask what happens if we allow non-trivial multiplicative relations among the coordinates of of $P$. We now show that the uniformity part of conjecture fails, i.e., for fixed $d$ and $n$, the number of exceptional subspaces may grow as the point $P$ varies. Although this example is somewhat artificial, it shows that some condition on $P$ or its orbit is needed if one is to drop the assumption in Conjecture 1.2 that $\mathcal{O}_{\phi}(P)$ be Zariski dense in $\mathbb{P}^{n}$. 
Proposition 6.1. Let $\phi_{d}: \mathbb{P}^{n} \rightarrow \mathbb{P}^{n}$ be the $d^{\text {th }}$-power map (4.1), let $\ell \geq 3$ be a prime such that $d$ is a primitive root modulo $\ell$, let $\zeta_{\ell}$ denote a primitive $\ell^{\text {th }}$-root of unity, let

$$
P=\left[1, \zeta_{\ell}, \alpha_{2}, \ldots, \alpha_{n}\right]
$$

be a point with $\alpha_{2}, \ldots, \alpha_{n}$ multiplicatively independent, and let $V$ be the (reducible) hypersurface

$$
V=\left\{\mathrm{x} \in \mathbb{P}^{n}: \frac{x_{1}^{\ell}-x_{0}^{\ell}}{x_{1}-x_{0}}=0\right\}
$$

Then for every $0<i<\ell$, the hyperplane

$$
H_{i}=\left\{\mathbf{x} \in \mathbb{P}^{n}: x_{1}=\zeta_{\ell}^{i} x_{0}\right\} \subset \mathbb{P}^{n}
$$

is an exceptional hyperplane for the map $\phi_{d}$.

Proof. It is clear that $V$ is the union of the $H_{i}$ for $0<i<\ell$. We also observe that

$$
\phi^{n}(P)=\left[1, \zeta_{\ell}^{d^{n}}, \alpha_{2}^{d^{n}}, \ldots, \alpha_{n}^{d^{n}}\right] \in H_{i} \Longleftrightarrow d^{n} \equiv i \quad(\bmod \ell) .
$$

Since $d$ is a primitive root modulo $\ell$, there is a unique integer $0<n_{i}<\ell$ such that

$$
d^{n} \equiv i \quad(\bmod \ell) \Longleftrightarrow n \equiv n_{i} \quad(\bmod \ell-1) .
$$

Thus each $H_{i}$ contains infinitely many points of $\mathcal{O}_{\phi_{d}}(P)$, and the multiplicative independence of $\alpha_{2}, \ldots, \alpha_{n}$ implies that $\mathcal{O}_{\phi_{d}}(P) \cap H_{i}$ is Zariski dense in $H_{i}$. Therefore $H_{i}$ is an exceptional subspace for $\phi_{d}$ and $P$.

Corollary 6.2. If we drop the assumption in Theorem 4.2 that $\mathcal{R}(P)=0$, then there does not exist a bound for $\# \mathcal{L}_{\phi, P}^{n}$ that depends only on $n$ and $d$, independent of the point $P$.

Proof. Proposition 6.1 says that for every prime $\ell$ such that $d$ is a primitive root modulo $\ell$, we can find a point $P_{\ell}$ such that

$$
\# \mathcal{L}_{\phi_{d}, P_{\ell}}^{n} \geq \ell-1 .
$$

To prove the corollary, it suffices to note that there exist many integers $d$ with the property that they are primitive roots for infinitely many primes $\ell$. See for example $[13,14]$, where it is proven that such $d$ exist, and indeed are quite common. (Of course, Artin's conjecture says that aside from the obvious exceptions, every $d$ has this property.)

On the other hand, we are able to show by a detailed case-by-case analysis that the conjecture holds for some choices of $P$ for which the relation set is non-trivial, i.e., for which the orbit $\mathcal{O}_{\phi}(P)$ is not Zariski dense. The following example demonstrates how such results are proven, while also illustrating the case-by-case analysis that makes it difficult to prove a general theorem.

Proposition 6.3. Let $\phi: \mathbb{P}^{3} \rightarrow \mathbb{P}^{3}$ be the $d^{\text {th }}$-power map (4.1), and let

$$
P=\left[\alpha_{0}, \alpha_{1}, \alpha_{2}, \alpha_{3}\right] \in \mathbb{P}^{3} \quad \text { satisfy } \quad \alpha_{0} \alpha_{1}=\alpha_{2} \alpha_{3},
$$


so $P$ lies on the quadric surface

$$
V=\left\{\left[x_{0}, x_{1}, x_{2}, x_{3}\right] \in \mathbb{P}^{3}: x_{0} x_{1}=x_{2} x_{3}\right\} .
$$

Assume further that $\mathcal{O}_{\phi}(P)$ is Zariski dense in $V$. Then for all $r \geq 1$, the set of exceptional linear spaces $\mathcal{L}_{\phi, P}^{r}$ is finite, and $\# \mathcal{L}_{\phi, P}^{r}$ may be bounded solely in terms of $n$ and $d$.

Proof Sketch. We note that in our notation, the assumption that $\overline{\mathcal{O}_{\phi}(P)}=V$ is equivalent to the assumption that the relation set $\mathcal{R}(P)$ has rank 1 and is generated by $(1,1,-1,-1)$. Since $P$ is not preperiodic and does not lie on any coordinate hyperplane, much of the proof of Theorem 4.2 carries over with no change. To complete the proof of Proposition 6.3, it remains to show that for any partition $\mathcal{I}_{p}$ that is not exceptional, the map

$$
\boldsymbol{F}: \mathcal{M}_{P} \longrightarrow \prod_{p \in \mathcal{P}_{r, n}} \prod_{T \in \mathcal{I}_{p}} \mathbb{P}^{\# T-1}, \quad \mathbf{m} \longmapsto\left(\left[u_{\sigma}(\mathbf{m})\right]_{\sigma \in T}\right)_{\substack{p \in \mathcal{P}_{r, n} \\ T \in \mathcal{I}_{p}}},
$$

is injective.

Let $\mathbf{m}, \widetilde{\mathbf{m}} \in \mathcal{M}_{P}$ be such that $\boldsymbol{F}(\mathbf{m})=\boldsymbol{F}(\widetilde{\mathbf{m}})$. This implies that for all $p \in \mathcal{P}_{r, n}$, all $T \in \mathcal{I}_{p}$, and all $\sigma, \tau \in T$, the vector

$$
v_{\sigma, \tau}=\left(k_{\sigma\left(p^{-1}(i)\right)}+\tilde{k}_{\tau\left(p^{-1}(i)\right)}-k_{\tau\left(p^{-1}(i)\right)}-\tilde{k}_{\sigma\left(p^{-1}(i)\right)}\right)_{0 \leq i \leq 3} \quad \text { is in } \mathcal{R}(P) .
$$

(If $i$ is not in the image of $p$, we set the $i^{\text {th }}$-coordinate of $v_{\sigma, \tau}$ to be 0 .) Since

$$
\mathcal{R}(P) \cap\left\{\left(e_{0}, e_{1}, e_{2}, e_{3}\right): e_{i}=0\right\}=\{\mathbf{0}\}
$$

for all $i$, if $r<n$ we can argue exactly as as in the proof of Theorem 4.6.

We now restrict to the case that $r=n$, so $\mathcal{P}_{r, n}=\{\mathrm{id}\}$. First we assume that there exists a $T \in \mathcal{I}$ and a $\sigma, \tau \in T$ such that $\sigma^{-1} \tau$ has no fixed points. A case-by-case analysis of the possibilities for $\sigma^{-1} \tau$ shows that (6.2) forces that $\mathbf{m}=\widetilde{\mathbf{m}}$. We illustrate with two cases.

Suppose that $\tau^{-1} \sigma=(0123)$. Since $\mathcal{R}(P)$ is generated by $(1,1,-1,-1)$, the fact that $v_{\sigma, \tau}$ is in $\mathcal{R}(P)$ implies that the second and third coordinates of $v_{\sigma, \tau}$ are negatives of one another, i.e.,

$$
k_{\sigma(1)}+\tilde{k}_{\tau(1)}-\tilde{k}_{\sigma(1)}-k_{\tau(1)}=-k_{\sigma(2)}-\tilde{k}_{\tau(2)}+\tilde{k}_{\sigma(2)}+k_{\tau(2)} .
$$

The assumption that $\tau^{-1} \sigma=(0123)$ implies that $\sigma(1)=\tau(2)$, which allows us to simplify (6.3) to

$$
\tilde{k}_{\tau(1)}-k_{\tau(1)}=\tilde{k}_{\sigma(2)}-k_{\sigma(2)} .
$$

Using $k_{i}=d^{m_{i}}$ and Lemma 4.7 as usual, we conclude that $k_{\sigma(2)}=\tilde{k}_{\sigma(2)}$ and $k_{\tau(1)}=$ $\tilde{k}_{\tau(1)}$. Substituting this into the relation equation and using the fact that the first two coordinates of $v_{\sigma, \tau}$ are the same, we find that $k_{\sigma(3)}=\tilde{k}_{\sigma(3)}$ and $k_{\sigma(1)}=\tilde{k}_{\sigma(1)}$. This argument works, mutatis mutandis, if $\tau^{-1} \sigma$ is any four cycle, as well as when $\tau^{-1} \sigma$ is either (02)(13) or (03)(12).

Next suppose that $\tau^{-1} \sigma=(01)(23)$. This choice of $\tau^{-1} \sigma$ means that

$$
\sigma(0)=\tau(1), \quad \sigma(1)=\tau(0), \quad \sigma(2)=\tau(3), \quad \sigma(3)=\tau(2) .
$$


Substituting these into the definition (6.3) of $v_{\sigma, \tau}$, we see that the vector $v_{\sigma, \tau}$ has the form $(X,-X, Y,-Y)$. But $\mathcal{R}(P)$ is generated by $(1,1,-1,-1)$, so $v_{\sigma, \tau}=\mathbf{0}$, which implies as usual that $\mathbf{k}=\tilde{\mathbf{k}}$.

We are left to consider the case that for all $T \in \mathcal{I}$ and for all $\sigma, \tau \in T$, there is an $i$, depending on $\sigma, \tau$, such that $\sigma(i)=\tau(i)$. This implies that the $i^{\text {th }}$-component of $v_{\sigma, \tau}$ is 0 , and so $v_{\sigma, \tau}$ must be the zero vector. Hence if there is a $t$ such that $m_{t} \neq \widetilde{m}_{t}$, then $\tau^{-1}(t)=\sigma^{-1}(t)$ for all $T \in \mathcal{I}$ and for all $\sigma, \tau \in T$. This says that $\mathcal{I}$ is exceptional, which completes the proof.

Remark 6.4. The proof of Proposition 6.3 gives a more general result, namely that there is a uniform bound for $\# \mathcal{L}_{\phi, P}^{r}$ provided that for every choice $H_{1}, \ldots, H_{n-r}$ of $n-r$ coordinate hyperplanes, the relation set satisfies

$$
\mathcal{R}(P) \cap H_{1} \cap \cdots \cap H_{n-r}=\{\mathbf{0}\} .
$$

\section{Acknowledgments}

The authors would like to thank the referee for his/her helpful suggestions. The first author's research supported by NSF DMS-0854755. The second author's research supported by NSF DMS-1002933.

\section{References}

[1] F. Amoroso and E. Viada, Small points on subvarieties of a torus, Duke Math. J. 150(3) (2009), 407-442.

[2] J.P. Bell, D. Ghioca and T.J. Tucker, The dynamical Mordell-Lang problem for étale maps, Amer. J. Math. 132(6) (2010), 1655-1675.

[3] R. Benedetto, D. Ghioca, P. Kurlberg and T. Tucker, A case of the dynamical Mordell-Lang conjecture, Mathematische Annalen (24 December 2010 (online)) 1-26. doi: 10.1007/s00208-0100621-4.

[4] L. Denis, Géométrie et suites récurrentes, Bull. Soc. Math. France 122(1) (1994), 13-27.

[5] J.-H. Evertse, Linear equations with unknowns from a multiplicative group whose solutions lie in a small number of subspaces, Indag. Math. (N.S.) 15(3) (2004), 347-355.

[6] J.-H. Evertse, K. Györy, C.L. Stewart and R. Tijdeman, On S-unit equations in two unknowns, Invent. Math. 92(3) (1988), 461-477.

[7] J.-H. Evertse, H.P. Schlickewei and W.M. Schmidt, Linear equations in variables which lie in a multiplicative group, Ann. Math. (2) 155(3) (2002), 807-836.

[8] G. Faltings, Diophantine approximation on abelian varieties, Ann. Math. (2) 133(3) (1991), 549-576.

[9] - The general case of S. Lang's conjecture, in Barsotti Symposium in Algebraic Geometry (Abano Terme, 1991), Perspective in Mathematics 15, 175-182, Academic Press, San Diego, CA (1994).

[10] D. Ghioca and T. Tucker, Periodic points, linearizing maps, and the dynamical Mordell-Lang problem, J. Number Theory 129(6) (2009), 1392-1403.

[11] D. Ghioca and T.J. Tucker, A dynamical version of the Mordell-Lang conjecture for the additive group, Compos. Math. 144(2) (2008), 304-316.

[12] D. Ghioca, T.J. Tucker and M.E. Zieve, Intersections of polynomials orbits, and a dynamical Mordell-Lang conjecture, Invent. Math. 171(2) (2008), 463-483.

[13] R. Gupta and M.R. Murty, A remark on Artin's conjecture, Invent. Math. 78(1) (1984), 127-130.

[14] D.R. Heath-Brown, Artin's conjecture for primitive roots, Q. J. Math. Oxford Ser. (2) 37(145) (1986), 27-38.

[15] M. Laurent, Équations diophantiennes exponentielles, Invent. Math. 78(2) (1984), 299-327. 
[16] G. Rémond, Borne générique pour le problème de Mordell-Lang, Manuscripta Math. 118(1) (2005), 85-97.

[17] H.P. Schlickewei and C. Viola, Generalized Vandermonde determinants, Acta Arith. 95(2) (2000), 123-137.

[18] P. Vojta, Siegel's theorem in the compact case, Ann. Math. (2) 133(3) (1991), 509-548.

Mathematics Department, Brown University, Box 1917, Providence, RI 02912, USA E-mail address: jhs@math.brown.edu

$U R L$ : www. math. brown. edu/ jhs

Mathematics Department, Brown University, Box 1917, Providence, RI 02912, USA

E-mail address: bviray@math.brown.edu

$U R L$ : www. math. brown.edu/ bviray 